\title{
Erratum to: Comparative metabolomics of root nodules infected with Frankia sp. strains and uninfected roots from Alnus glutinosa and Casuarina cunninghamiana reflects physiological integration
}

\author{
James M. Brooks ${ }^{1}$ - David R. Benson ${ }^{1,2}$
}

Published online: 21 April 2016

(C) Springer Science+Business Media Dordrecht 2016

Erratum to: Symbiosis

DOI 10.1007/s13199-016-0379-x

In Brooks and Benson (Comparative metabolomics of root nodules infected with Frankia sp. strains and uninfected roots from Alnus glutinosa and Casuarina cunninghamiana reflects physiological integration. Symbiosis doi:10.1007/s13199016-0379-x), Fig. 1a and b were reversed, and in Fig. 3 the letters "a" and "b" were omitted. The corrected version is online.

The online version of the original article can be found at http://dx.doi.org/ 10.1007/s13199-016-379-x.

David R. Benson

david.benson@uconn.edu

1 Department of Molecular and Cell Biology, University of Connecticut, U-3125, Storrs, CT 06279, USA

2 Present address: Biology Department, Charleston Southern University, 9200 University Boulevard, Charleston, SC 29406, USA 
Fig. 1 Box plot pairs of amino acid levels in roots from uninfected plants, and root nodules of A. glutinosa (a) and C. cunninghamiana (b). For comparisons of relative abundances, amino acids are ordered from the most to the least abundant in C. cunninghamiana. The first box in each pair represent uninfected roots; the second box in each pair represents nodules. The boxes show the interquartile range, and bars indicate data points below the first and above the third quartile. Lines in the boxes are the median value. Filled red boxes denote a significant increase (Student's ttest $p<0.05 ; n=6$ ) in nodules versus roots, and filled green boxes denote a significant decrease in nodules versus roots. Gray boxes indicate no significant change J. M. Brooks, D. R. Benson
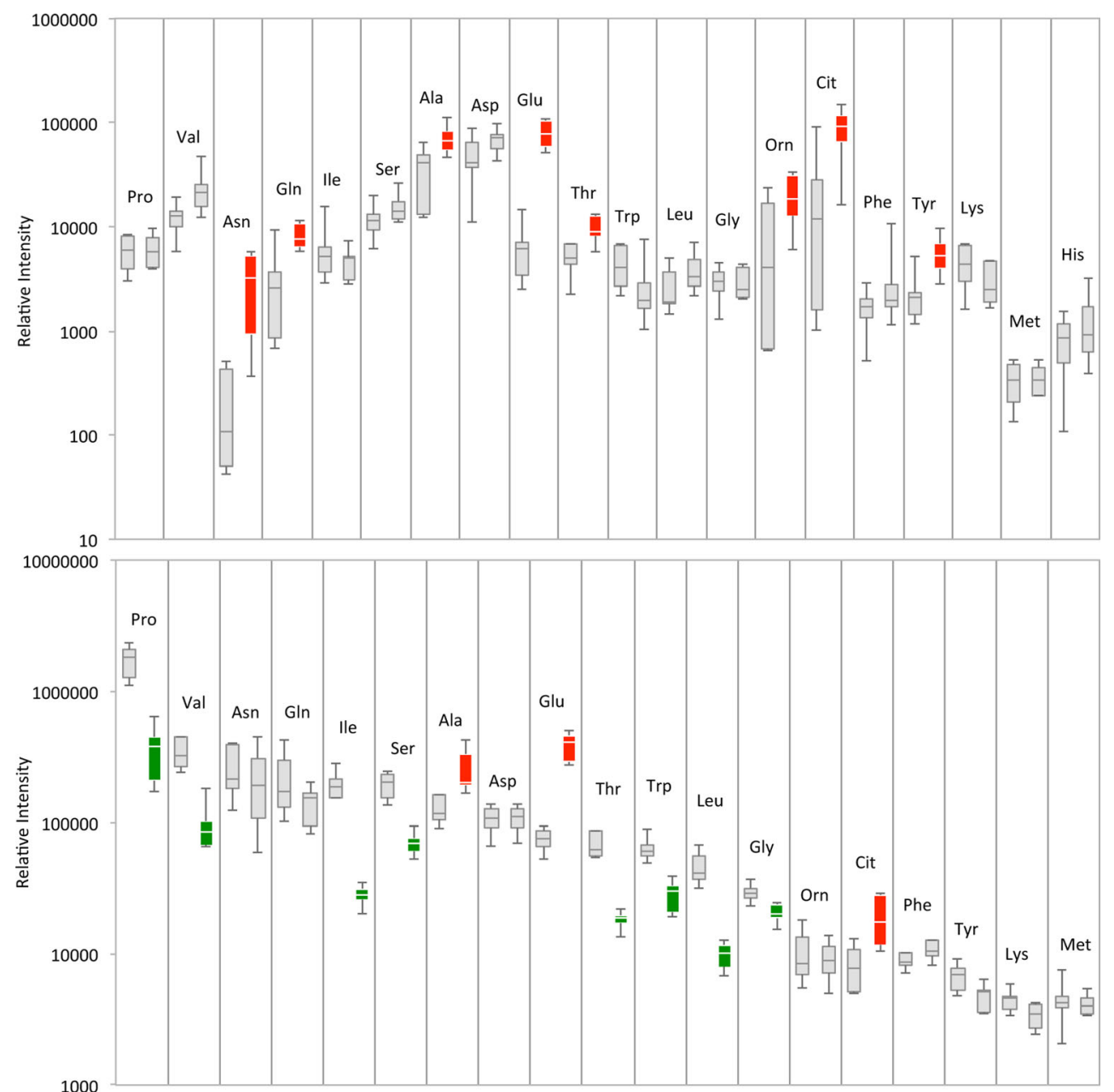
Fig. 3 Box plot pairs of relative sugar levels in roots from uninfected plants, and root nodules of A. glutinosa (a) and C. cunninghamiana (b). The first box in each pair denotes uninfected roots; the second box in each pair denotes nodules. Data statistics and presentation are as in Fig. 1
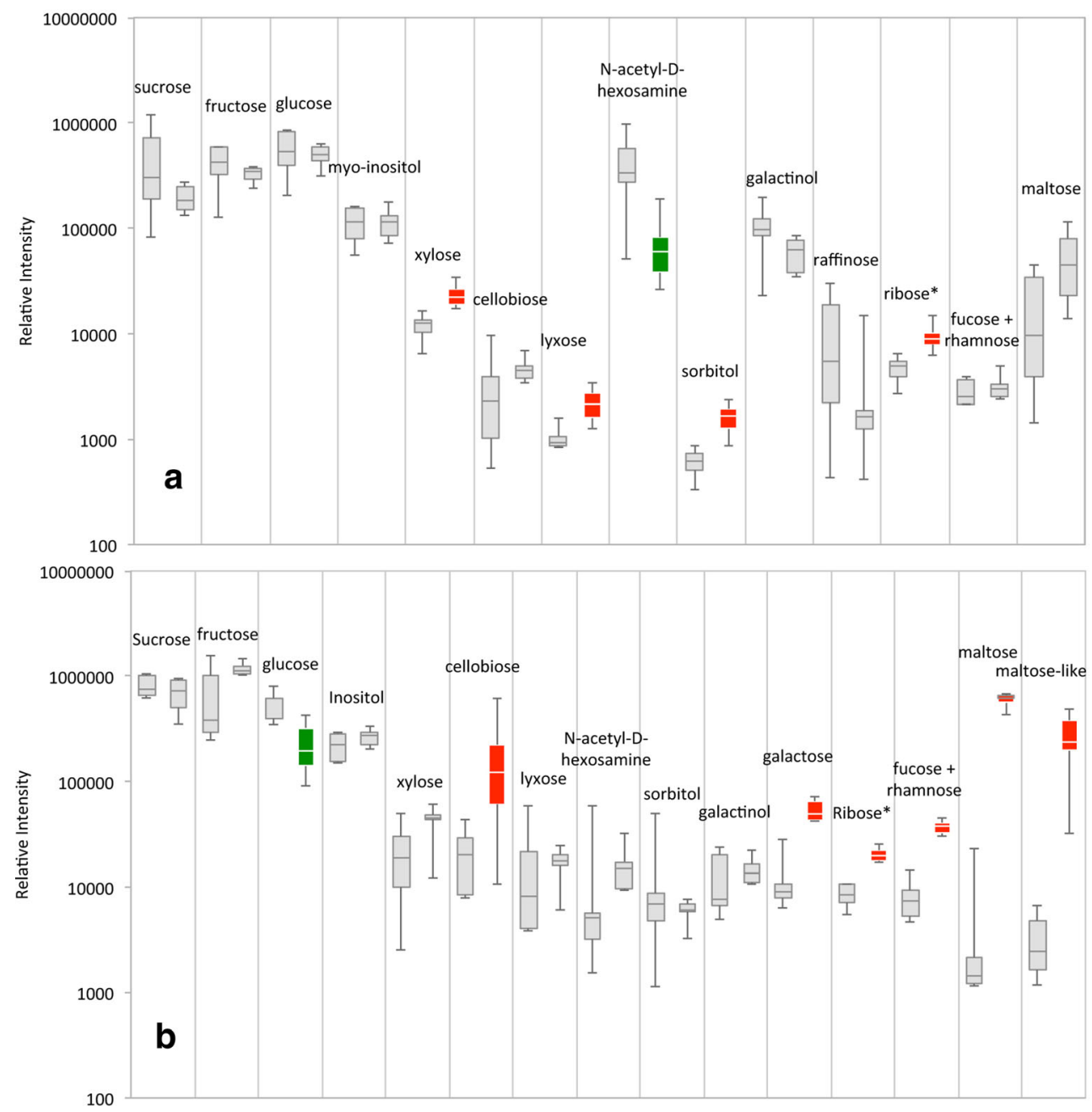